\title{
Desarrollo y validación de un inmunoensayo para la detección de la leptina caprina\#
}

\author{
Development and validation of an immunoassay for the detection of caprine leptin
}

\author{
J Campuzano-Granados ${ }^{\mathrm{a}}$, AJ Vallecillo-Maza ${ }^{\mathrm{b}}$, M Sierra-García ${ }^{\mathrm{c}}$, E Rodríguez-Fores ${ }^{\mathrm{b}}$, \\ C Parada-Colin ${ }^{b}$, C Espitia ${ }^{b}$, G Perera-Marín $^{\mathrm{d}}$, IE Candanosa-Aranda ${ }^{\mathrm{c}^{*}}$ \\ aDepartamento de Patología, Facultad de Medicina Veterinaria y Zootecnia, Universidad Nacional Autónoma de México, \\ México DF, México. \\ bepartamento de Inmunología, Instituto de Investigaciones Biomédicas, Universidad Nacional Autónoma de México, \\ México DF, México. \\ ${ }^{\mathrm{c}}$ Centro de Enseñanza, Investigación e Extensión de Producción Animal en Altiplano, Facultad de Medicina Veterinaria y Zootecnia, \\ Universidad Nacional Autónoma de México, Tequisquiapan, México. \\ ${ }^{\text {d} D e p a r t a m e n t o ~ d e ~ R e p r o d u c c i o ́ n, ~ F a c u l t a d ~ d e ~ M e d i c i n a ~ V e t e r i n a r i a ~ y ~ Z o o t e c n i a, ~ U n i v e r s i d a d ~ N a c i o n a l ~ A u t o ́ n o m a ~ d e ~ M e ́ x i c o, ~}$ \\ México DF, México.
}

\begin{abstract}
SUMMARY
Leptin is a protein hormone synthesised and secreted by white adipocytes. This hormone is involved in various metabolic processes, energy balance, fertility, inflammation and angiogenesis. In order to have a system for determining the concentration of leptin in goats, in this work we developed and validated an immunodiagnostic system (ELISA) using goat leptin obtained in recombinant form in Escherichia coli. Polyclonal antibodies were prepared in rabbits and a specific ELISA system was developed, then the binding capacity of the recombinant leptin specific to the receptor in the central nervous system was demonstrated by immunohistochemistry. Prior to the development of immunometric system, reagents were evaluated in a commercial RIA standardised system, and results indicated support for the evaluation system. The displacement curve of recombinant leptin showed no difference in the slope or the correlation coefficient with reference leptin, although the IC50 dose was lower. Binding capacity of the recombinant protein to specific receptors in the hypothalamus was identified. No significant differences in plasma concentrations of leptin obtained by ELISA and those obtained in the study using the RIA formulated for ruminants were obtained. These observations suggest that the developed ELISA could be an effective method for determining the concentration of goat serum leptin.
\end{abstract}

Key words: immunoassay, leptin, goat, ELISA.

\section{RESUMEN}

La leptina es una hormona proteica sintetizada y secretada por los adipocitos blancos, que controla varios procesos metabólicos, balance energético, fertilidad, inflamación y angiogénesis. Este estudio muestra el desarrollo y validación de un sistema de inmunodiagnóstico (ELISA) usando la leptina de cabra recombinante generada en Escherichia coli, a partir de esta se generó su correspondiente anticuerpo policlonal en conejos. Previo al desarrollo del sistema se demostró por inmunohistoquímica la capacidad de unión de la leptina recombinante específica a su receptor en el sistema nervioso central. Adicionalmente, el antígeno y el anticuerpo generados se evaluaron en un sistema normalizado de RIA comercial. Para validar el ELISA se comparó las concentraciones plasmáticas de leptina obtenidas con el ELISA en estudio y con un RIA para rumiantes. La curva de desplazamiento de la leptina recombinante no mostró diferencia en su pendiente ni en el coeficiente de correlación con la leptina de referencia, aunque la dosis $\mathrm{IC}_{50}$ fue menor. $\mathrm{Se}$ demostró la capacidad de unión de la proteína recombinante a receptores específicos del hipotálamo caprino. No hubo diferencias significativas en las concentraciones plasmáticas de leptina obtenidas por el ELISA en estudio y aquellas obtenidas utilizando el RIA formulado para rumiantes. Los resultados sugieren que el ELISA desarrollado podría ser un método eficaz para determinar la concentración de leptina en suero de cabra.

Palabras clave: inmunoensayo, leptina, cabra, ELISA.

\section{INTRODUCCIÓN}

La leptina es una proteína de 167 aminoácidos sintetizada y secretada en el adipocito blanco, que provee una señal de retroalimentación entre el tejido adiposo y sus receptores en el hipotálamo (Henry y col 2008). Se ha sugerido que

\footnotetext{
Aceptado: 30.04.2015.

\# Financiamiento: PAPIIT IN216407 DGAPA-UNAM.

* ieca@unam.mx
}

la leptina en calostro y leche puede actuar en la fisiología del neonato antes y después de su absorción digestiva. Un estudio en cabras menciona que las concentraciones de leptina en leche están relacionadas con el peso y la condición corporal de la madre y su cría. Sin embargo, los posibles efectos de leptina en el crecimiento de los cabritos se desconocen (Magistrelli y col 2008).

Las primeras leptinas recombinantes en producirse comercialmente fueron de origen humano (Altmann y col 1995) y murino (Campfield y col 1995). Para determinar la concentración de la leptina, generalmente se emplea la 
técnica de radioinmunoensayo (RIA). Aunque esta herramienta analítica es altamente específica, sensible y de bajo costo por muestra, tiene como desventaja que requiere equipo y personal altamente especializado, licencias para el manejo y desecho de productos radiactivos (Ehrhardt y col 2000). Actualmente la determinación de las distintas hormonas se realiza por métodos colorimétricos, siendo la técnica de ELISA la más comúnmente utilizada (Chow y col 2003). Ejemplo de ello es que se cuenta con un estuche comercial de inmunodiagnóstico multiespecie (ELISA, XL-85K; Linco, St Louis MO, USA) para esta proteína, así como el estuche de inmunodiagnóstico ELISA para determinar la leptina sérica en cabra (ELISA kit for leptin [LEP], E9004Cp, Life science Inc.). Sin embargo, el elevado costo de este producto hace que su uso en México se limite a trabajos de investigación, lo que limita el estudio del papel que esta hormona juega en el balance energético y sus repercusiones en situaciones clínico-patológicas y en el análisis de los aspectos reproductivos de las cabras.

El objetivo del estudio consistió en desarrollar y validar un sistema de inmunodiagnóstico ELISA para la determinación de la concentración sérica de la leptina caprina. Esto, produciendo una leptina caprina recombinante, caracterizándola inmunológicamente, y validando su actividad biológica mediante la determinación de su capacidad de unión a los receptores presentes en el sistema nervioso central. La validación del sistema se determinó con sueros caprinos previamente evaluados en el sistema de RIA de rumiantes (Delavaud y col 2000).

\section{MATERIAL Y MÉTODOS}

\section{DISEÑO DE LA PROTEÍNA RECOMBINANTE HIS TAG-GLEPTIN}

La proteína recombinante His tag-gLeptin se obtuvo a partir de los oligonucleótidos diseñados LEPTIN2F 5'-(GCATATGGTGCCCATCCGCAAGG)-3' y LEPTIN2R 5' - (GGGATCCTAGCACCCAGGGCTGAGG) -3' de acuerdo con la secuencia reportada de Capra hircus obese gene (GenBank accession number: AM114397.2). Para la clonación direccionada del vector de expresión procarionte pET15b (Novagen, Darmstadt, Germany) se utilizaron las enzimas de restricción NdeI y BamHI que actuaron sobre LEPTIN2F y LEPTIN2R, respectivamente (secuencias subrayadas). Se estimó un peso molecular de 18,4 kDa utilizando WinPep 7 (Dynojet, Nevada, USA).

\section{EXTRACCIÓN DE RNA DE TEJIDO GRASO PERIRRENAL DE CABRA}

La extracción de RNA se realizó en un gramo de tejido graso mediante el procedimiento descrito por Chomczynski y Sacchi (1987). La cuantificación del RNA se determinó por el método de absorbancia en la región ultravioleta, con la relación 260/280 con el empleo del nanodrop, y para verificar la calidad del RNA total se realizó una electroforesis en gel de agarosa-formaldehído.

\section{AMPLIFICACIÓN POR RT-PCR DE LA SECUENCIA} CODIFICANTE

El cDNA se obtuvó a partir de $5 \mu \mathrm{g}$ de RNA tratado con DNAsa I (Invitrogen). La reacción de síntesis se realizó en un volumen de $20 \mu \mathrm{l}$ que contenía $0,5 \mathrm{mM}$ de dNTP's, 8 pmoles del primer LEPTIN2R y $100 \mathrm{U}$ de la transcriptasa reversa (SuperScript III, Invitrogen). La reacción se incubó por $1 \mathrm{~h}$ a $42{ }^{\circ} \mathrm{C}$ y después de inactivar la enzima a $70{ }^{\circ} \mathrm{C}$ por $15 \mathrm{~min}$ se procedió a realizar la amplificación por PCR. La reacción se llevó a cabo en un volumen de $25 \mu \mathrm{l}$ que contenía $50 \mathrm{mM}$ de cada dNTP, 2 pmoles de cada oligonucleótido (LEPTIN2F y LEPTIN2R), $2 \mu \mathrm{l}$ de cDNA y $1.25 \mathrm{U}$ de Taq DNA polimerasa (Invitrogen, Foster city, USA). La reacción se incubó durante 2 min a $94{ }^{\circ} \mathrm{C}$ y cada ciclo de amplificación consistió de $40 \mathrm{~s}$ a $94{ }^{\circ} \mathrm{C}$, alineamiento por $25 \mathrm{~s}$ a $66{ }^{\circ} \mathrm{C}$, extensión por $50 \mathrm{~s}$ a $72{ }^{\circ} \mathrm{C}$; y una extensión final a $72{ }^{\circ} \mathrm{C}$ por $5 \mathrm{~min}$. Una muestra de $4 \mu \mathrm{l}$ se colocó en un gel de agarosa al $1 \%$ para identificar la amplificación. Se observó una banda a la altura de $\pm 450 \mathrm{bp}$. El producto esperado correspondió a 454 bp.

\section{CLONACIÓN Y EXPRESIÓN DE LA LEPTINA DE CABRA RECOMBINANTE EN Escherichia coli}

El producto de PCR de la secuencia codificante de la leptina se clonó en el vector pCRII (TOPO TA, Invitrogen, Foster city, USA) siguiendo las instrucciones del fabricante. Una de las clonas que contenía el inserto se secuenció de manera automática (Unidad de síntesis y secuenciación de DNA, Instituto de Biotecnología-UNAM), de esta misma clona se subclonó en el plasmido pET15b (Novagen Darmstadt, Germany) empleando los sitios de restricción NdeI-BamHI. Con la construcción obtenida se transformó la cepa de E. coli Rosetta (DE3).

\section{EXPRESIÓN Y PURIFICACIÓN DE LA LEPTINA DE CABRA}

La síntesis de la proteína recombinante (pET15bgLeptin) por la cepa Rosetta (DE3) se llevó a cabo mediante la aplicación de IPTG (Isopropyl $\beta$-D-1thiogalactopyranoside) a una concentración final de 250 $\mu \mathrm{M}$. La proteína recombinante se obtuvo previa disolución de los cuerpos de inclusión en amortiguador Tris- $\mathrm{HCl}$ (10 mM), pH 8,0 que contenía $8 \mathrm{M}$ de urea y $50 \mathrm{mM}$ de $\mathrm{NaCl}$ (12 h a $4{ }^{\circ} \mathrm{C}$ en agitación constante). Una vez solubilizada, la proteína se purificó en una cromatografía de afinidad (HiTrap chelating, Amersham pharmacia) con el empleo del equipo AKTA FPLC. Las fracciones colectadas se monitorearon a $280 \mathrm{~nm}$ y los picos de proteína identificados se dializaron con amortiguador 
Tris- $\mathrm{HCl}(10 \mathrm{mM}), \mathrm{pH} 8,0$, que contenía $50 \mathrm{mM}$ de $\mathrm{NaCl}$ y cantidades decrecientes de urea $(4 ; 2 ; 1,0,5 ; 0,25$ y 0,0 $\mathrm{M})$. Para cada una de las concentraciones decrecientes de urea, la diálisis se realizó a $4{ }^{\circ} \mathrm{C}$ durante $8 \mathrm{~h}$ con cambios cada $4 \mathrm{~h}$. Para favorecer la renaturalización de la leptina la diálisis sin urea se repitió dos veces. Al término, la proteína se alicuoteó y almacenó a $-20{ }^{\circ} \mathrm{C}$ hasta su uso (Nilsson y col 1997).

\section{SDS-PAGE E INMUNOTRANSFERENCIA}

El peso molecular de la proteína recombinante purificada se analizó en geles de poliacrilamida de acuerdo con el método descrito por Laemmli (1970). Se utilizó un gel al 12\%, porcentaje cercano a lo descrito por Kling (2009) para leptina de salmón. La proteína presente en el gel se transfirió a una membrana de PVDF e incubó con anti-gLeptina, 1:500 durante 24 h y luego la membrana se incubó por $2 \mathrm{~h}$ con un anticuerpo secundario (anti-rabbit IgG acoplado a fosfatasa alcalina) 1:20.000. La banda de proteína inmunorreactiva se reveló con 1 $\mathrm{ml}$ de azul de tetrazolium en dimetilformamida y $1 \mathrm{ml}$ de 5-bromo-4-cloro-3-indolilfosfato en DMF disuelto en $100 \mathrm{ml}$ PBS.

Para la capacidad de unión de la proteína recombinante a receptores específicos localizados en el hipotálamo se emplearon cortes histológicos del hipotálamo, estos se incubaron con el anticuerpo antihistidina como control negativo, mientras que la unión de la leptina recombinante a los receptores se observó cuando se incubó previamente con la leptina recombinante y después con el anticuerpo antihistidina.

\section{PRODUCCIÓN DE ANTICUERPOS POLICLONALES DE LEPTINA RECOMBINANTE}

El antisuero de la leptina recombinante de cabra se generó en conejos hembras de la raza Nueva Zelanda de acuerdo con lo descrito por Gertler (1998) con algunas modificaciones. En breve, cada animal recibió en la región dorsal por vía subcutánea un volumen de $100 \mu \mathrm{l}$, que contenía $100 \mu \mathrm{g}$ de proteína emulsificada con aceite mineral y manoleato (adyuvante incompleto de Freund's; Sigma, St Louis, MO, USA). La aplicación de la hormona se realizó en cuatro ocasiones con intervalos de 15 días entre ellas. Una quinta aplicación se realizó por vía intradérmica con hormona solubilizada en PBS, pH 7,4. Una vez concluido el esquema de inyección, los conejos se sangraron 2 semanas después. Aunque con el esquema de inmunización desarrollado se obtuvieron sueros con títulos de anticuerpos bajos (1:200), se consideraron para su purificación en una cromatografía de afinidad (proteína G-sepharosa, Pharmacia) y la IgG obtenida se marcó con peroxidasa de rábano (Porstmann y col 1985) (EZ-Link Plus Activated peroxidase, Pierce Biotechnology) de acuerdo a las especificaciones del fabricante.

\section{CURVA DE DESPLAZAMIENTO}

Con el objeto de analizar la actividad inmunológica de la leptina caprina recombinante se compararon las curvas de desplazamiento generadas por la leptina humana del sistema comercial de radioinmunoensayo multiespecie (Multispecies Leptin RIA Kit, Linco, Research Inc, St. Charles, MO, USA) con aquella que produjo la leptina recombinate de cabra de nuestro estudio. Para esto la leptina recombinante de cabra se resuspendió en el amortiguador del ensayo (PBS 0.05 M, pH 7,4 EDTA, 0.025 M, azida de sodio $0,08 \%$, triton $\mathrm{X} 100,0,05 \%$ y BSA $1 \%$ ) a las dosis de 0,$5 ; 1,0 ; 2,5 ; 5,0 ; 10,0$ y 20,0 ng de proteína por $\mathrm{ml}^{-1} \mathrm{y}$ ambas muestras se sometieron a las condiciones de análisis descritas por el sistema. Con este estudio se calculó la pendiente de cada curva, la dosis de inhibición de proteína inmunorreactiva al $50 \%$ de la relación $\mathrm{B} / \mathrm{Bo}$ de curva dosis-respuesta (IC50) y adicionalmente se calculó la sensibilidad basada en la cantidad mínima detectable de proteína inmunorreactiva al 85\% (Matteri y col 1986).

\section{ELISA DE LEPTINA}

Una vez que se caracterizó la leptina recombinante caprina y se obtuvo su anticuerpo, se procedió al desarrollo del sistema de inmunodiagnóstico. Para ello se desarrolló el ELISA por competencia utilizando placas de microtitulación de poliestireno de 96 pozos (Maxisorp, NUNC). El sistema se inició recubriendo cada pozo de la placa con anticuerpo primario (anti-Leptina, 1:2000) diluido en 100 $\mu \mathrm{l}$ de amortiguador de carbonatos $(0,05 \mathrm{M}, \mathrm{pH} 9,6)$ durante 12 h a $4{ }^{\circ} \mathrm{C}$. Al término, el exceso de anticuerpo se retiró con tres lavados de fosfatos (PBS $0.05 \mathrm{M})$ que contenía 0,05\% de Tween-20 (PBS-T). Subsecuentemente, en el correspondiente pozo se colocó un volumen de $100 \mu \mathrm{l}$ de muestra de suero (1:5) o leptina caprina recombinante $(12,5$ a $0,048 \mathrm{ng} / \mathrm{ml})$ diluidos en PBS-T que contenían $1 \%$ de BSA (PBS-TB). Una vez que se depositó la muestra, la placa se incubó en una cámara húmeda a $4{ }^{\circ} \mathrm{C}$ durante 16 h. Al término, cada pozo se lavó tres veces con PBS-T y después recibió $100 \mu \mathrm{l}$ de PBS-TB que contenía el anticuerpo secundario anti-IgG de cabra conjugado con peroxidasa de rábano $(1,8 \mu \mathrm{g} / \mathrm{ml})$ y se continuó con la incubación durante 90 min a temperatura ambiente en agitación suave.

Al término, cada pozo se lavó tres veces con PBS-T y se inició el revelado colocando $100 \mu \mathrm{l}$ de una solución de PBS-T con $1 \%$ de peróxido de hidrógeno y dihidrocloro tetrametilbenzidina (Sigma) y una incubación de $20 \mathrm{~min}$ a temperatura ambiente en obscuridad. La reacción concluyó añadiendo en cada pozo $50 \mu \mathrm{H}_{2} \mathrm{SO}_{4}$. La absorbancia fue determinada a $450 \mathrm{~nm}$ utilizando un lector de microplacas (Modulus $^{\mathrm{TM}}$ II, BioSystem, Inc) (Hardie y col 1996).

La curva estándar se generó a partir de los datos de las lecturas de absorbancia de cada referencia estándar con su concentración correspondiente ( $\mathrm{ng} / \mathrm{ml}$ ). La concentración de leptina de cada muestra de suero fue determinada usando la 
absorbancia correspondiente y extrapolada a los valores de la curva estándar y multiplicada por el factor de dilución.

\section{VALIDACIÓN DEL SISTEMA}

Para validar el sistema de ELISA se comparó la concentración plasmática de leptina determinada en este estudio con aquellos valores obtenidos con el sistema de RIA homológo de doble anticuerpo en fase líquida para leptina ovina que también es eficiente para la determinación de leptina plasmática en bovinos y caprinos (Devalaud y col 2000). Este sistema de rumiantes utilizó como trazador y estándar de referencia a la leptina ovina recombinante, a partir de ella se generó su propio anticuerpo. Para esto se diseñó un estudio que permitió obtener la variación en la concentración plasmática de leptina esperable debido al crecimiento de las cabras. Se emplearon 18 cabritos castrados de la cruza Boer X Alpino francés, se sometieron a un sistema de pastoreo rotacional en praderas de alfalfa, Lolium perenne y Dactylis glomerata. Las muestras sanguíneas se tomaron de la vena yugular durante la mañana previa al pastoreo y el suero se obtuvo después de la centrifugación a $1.500 \mathrm{~g}$ de cada muestra. Las muestras se colectaron a partir del día 30 hasta los 180 días de edad, con un intervalo de muestreo cada 15 días. El análisis comparativo entre el sistema de RIA validado y el sistema de ELISA desarrollado en este estudio se determinó mediante análisis de varianza con un diseño completamente al azar con mediciones repetidas en el tiempo y un análisis de regresión lineal empleando el
PROC REG del paquete estadístico SAS versión 9.4 para Windows (SAS Inst. Inc, Cary NC, USA). Esto último, para obtener la relación existente entre las concentraciones plasmáticas de leptina obtenidas mediante nuestra leptina y del RIA anteriormente señalado.

\section{RESULTADOS Y DISCUSIÓN}

El análisis de la secuencia del ADNc de la leptina caprina obtenida en este estudio mostró una homología del $100 \%$ respecto de la secuencia del gen de leptina de cabra publicada en el gene bank. El producto de amplificación de RT-PCR para el gen de leptina caprina que se utilizó para la clonación y expresión de la proteína recombinante presentó el número de pares esperado (Kawachi y col 2007). El patrón electroforético de la leptina recombinante caprina en el gel de SDS-PAGE mostró una proteína de peso molecular de $16 \mathrm{kDa}$ y cuya banda fue reconocida por el antisuero policlonal de conejo y por el antisuero dirigido en contra del tallo de histidina. Este resultado coincidió con lo reportado para la leptina de salmónidos en donde la secuencia del gen de leptina de salmón del Atlántico (Atlantic salmon) dio origen a una proteína recombinante de 16 kDa utilizada para la cuantificación de leptina en suero de salmón (Kling y col 2009). Se verificó la capacidad de unión de la proteína recombinante a receptores específicos localizados en cortes histológicos de hipotálamo. Este resultado establece que la leptina recombinante tiene actividad biológica al reconocer a su receptor.

A

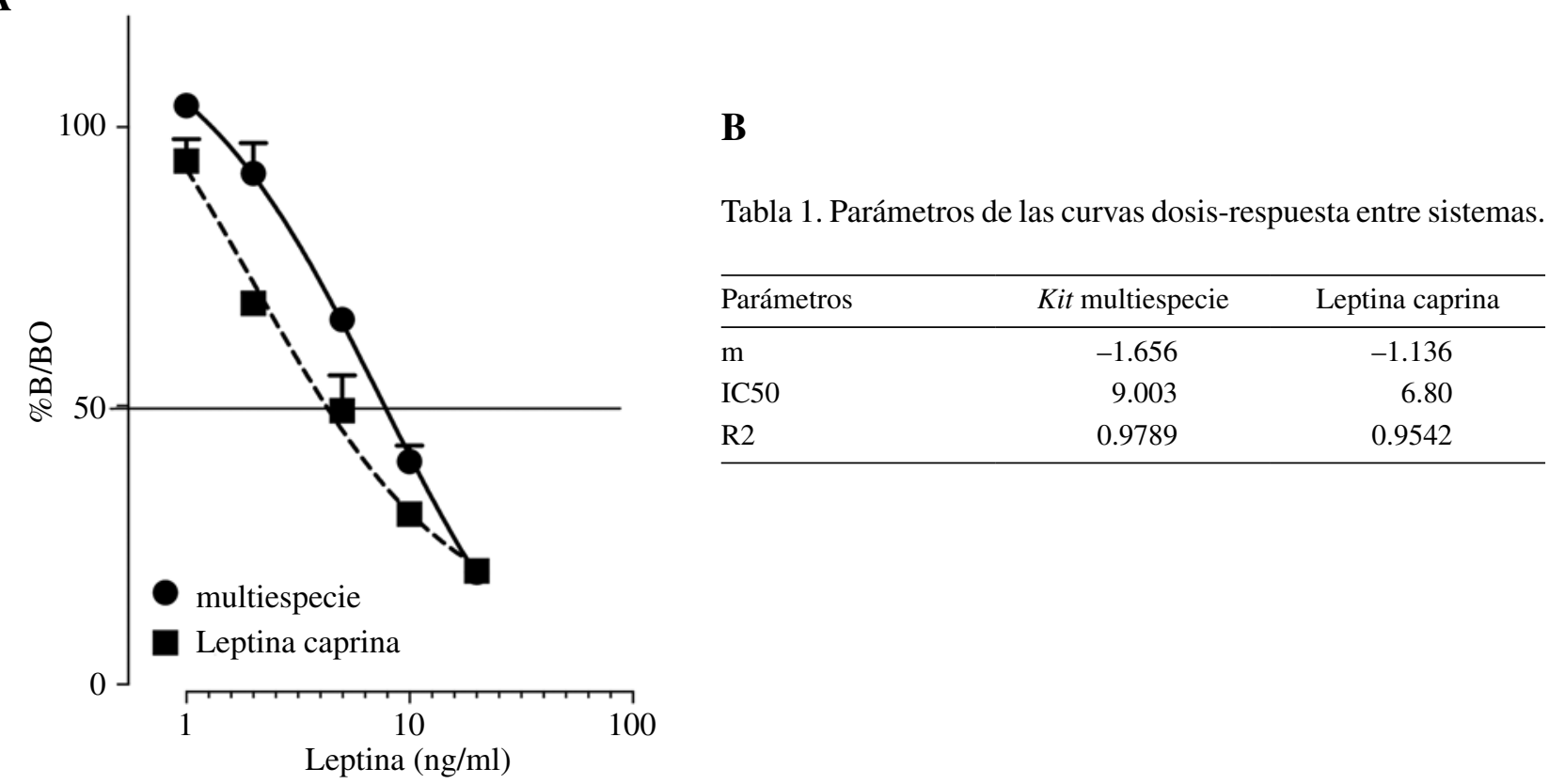

Figura 1. Patrón de la curva dosis-respuesta de la leptina caprina recombinante en el sistema de RIA multiespecie. Los puntos en cada curva representan el promedio de tres réplicas $\pm \mathrm{EE}$. La tabla resume los parámetros de cada curva.

Pattern of the curve dose -response recombinant caprine leptin in RIA multispecies system. The points on each curve represent the average of three replicates $\pm \mathrm{EE}$. The table summarises the parameters of each curve. 
El análisis de la curva dosis-respuesta generada a partir del porcentaje de B/Bo y el logaritmo de la dosis de la leptina recombinante caprina y del estándar de referencia de leptina humana, determinó que existe similitud en el valor de la pendiente y en la correlación entre ambas curvas, lo que demuestra la actividad inmunológica entre ambas hormonas. Sin embargo, la cantidad de proteína inmunorreactiva referida como las dosis $\mathrm{IC}_{50}$ fue significativamente menor $(\mathrm{P}<0,05)$, en la leptina caprina respecto de lo obtenido en la leptina humana. En otras palabras, fue necesaria una menor cantidad (figura 1) de leptina recombinante caprina para obtener el 50\% de inhibición del sistema respecto de la requerida de leptina humana $(4,98 \mathrm{ng} / \mathrm{ml}$ vs. $7,7 \mathrm{ng} /$ $\mathrm{ml}$, respectivamente). Este resultado es muy interesante considerando que diversos autores han descrito una alta homología entre la humana y la de rumiantes, en particular para la especie ovina (Delavaud y col 2000), por tanto este dato sugiere que el reconocimiento de los epítopes de la leptina caprina por el correspondiente anticuerpo está localizado en sitios distintos y esto podría explicar la mayor especificidad del anticuerpo multiespecie (guinea pig leptin antibody) por la leptina recombinante obtenida en este estudio (Gertler y col 1998). La sensibilidad al $85 \%$ de la relación $\mathrm{B} / \mathrm{Bo}$ de la curva dosis-respuesta fue de un valor absoluto de $1,0 \mathrm{ng} / \mathrm{ml}$ para la leptina caprina. Este valor fue similar al reportado para leptina ovina que correpondió a $0,83 \mathrm{ng} / \mathrm{ml}$ determinado al $95 \%$ de B/B0 (Delavaud y col 2000). En el presente estudio se evaluó el cambio en la concentración plasmática de leptina en cabras de 60 a 180 días, no encontrando un estudio similar para ser comparado. Sin embargo, bajo las condiciones ensayadas en este estudio la cantidad de proteína mínima detectable por el estándar humano correspondió a 3,0 ng/ml. Esta mayor sensibilidad que se observó con la leptina caprina como patrón de referencia en el kit multiespecie podría deberse a la alta afinidad por el anticuerpo. Finalmente, la validación del sistema ELISA señaló que la comparación de la concentración plasmática de leptina determinada con el ELISA en estudio y el RIA para rumiantes (Delavaud y col 2000) no presentaron diferencias significativas $(\mathrm{P} \leq 0,01)$, lo que sugiere que ambas proteínas de origen recombinante presentan una alta homología y los sitios de reconocimiento por sus correspondientes anticuerpos es similar.

Los anticuerpos empleados en ovino y bovino para evaluar la concentración de leptina se realiza por medio de RIA o ELISA en plasma, debido a la gran similitud en la composición de aminoácidos de leptina en estas dos especies (Delavaud y col 2000). Chilliard y col (2005) obtuvieron concentraciones plasmáticas tres o cuatro veces menores, empleando el kit RIA de leptina multiespecies, que utiliza leptina humana recombinante, respecto de lo obtenido con un RIA específico para rumiantes.

Basados en los resultados del presente trabajo podemos señalar que el ELISA caprino desarrollado es una herramienta inmunoanalítica que permitirá en un futuro próximo la evaluación de la leptina caprina bajo diferentes condiciones clínico-patológicas.

\section{AGRADECIMIENTOS}

Al Dr. Duane Keisler del Animal Science Research Center, College of Agriculture, Food and Natural Resources, University of Missouri, por la determinación de leptina sérica por RIA.

\section{REFERENCIAS}

Altmann SW, JC Timans, FL Rock, JF Bazan , RA Kastelein. 1995. Expression and purification of a synthetic human obese gene product. Protein Expr Purif 6, 722-726.

Campfield LA, FJ Smith, Y Guiez, R Devos, P Burn. 1995. Recombinant mouse $\mathrm{OB}$ protein: evidence for a peripheral signal linking adiposity and central neural networks. Science 269, 546-549.

Chilliard Y, M Bonnet, C Delavaud, Y Faulconnier, C Leroux, J Djiane, F Bocquier. 2001. Leptin in ruminants. Gene expression in adipose tissue and mammary gland, and regulation of plasma concentration. Domestic Anim Endocrinol 21, 271-295

Chomczynski P, N Sacchi. 1987. Single-step method of RNA isolation by acid guanidinium thiocyanate-phenol-chloroform extraction. Anal Biochem 162,156-159.

Chow VTK, MC Phoon. 2003. Measurement of serum leptin concentrations in university undergraduates by competitive ELISA reveals correlations with body mass index and sex. Adv Physiol 27, 70-77.

Delavaud C, F Bocquier, Y Chilliard, Keisler DH, A Gertler. 2000. Plasma leptin determination in ruminants: effect of nutritional status and body fatness on plasma leptin concentration assessed by a specific RIA in sheep. $J$ Endocrinol 165, 519-526.

Ehrhardt RA, RM Slepetis, J Siegal-Willott, ME van Amburgh, AW Bell, $\mathrm{R}$ Boisclair. 2000. Development of a specific radioimmunoassay to measure physiological changes of circulating leptin in cattle and sheep. J Endocrinol 166, 519-528.

Gertler A, J Simmons, DH Keisler. 1998. Large-scale preparation of biologically active recombinant ovine obese protein (leptin). FEBS Letters 422, 137-140.

Hardie LJ, DV Rayner, S Holmes, P Trayhurn. 1996. Circulating leptin levels are modulated by fasting, cold exposure, and insulin administration in lean but not Zucker ( $\mathrm{fa} / \mathrm{fa}$ ) rats as measured by ELISA. Biochem Biophys Res Comm 223, 660-665.

Henry BA, IJ Clarke. 2008. Adipose tissue hormones and the regulation of food intake. J Neuroendocrinol 20, 842-849.

Kawachi H, SH Yang, A Hamano, T Matsui, SB Smith, H Yano. 2007. Molecular cloning and expression of bovine (Bos taurus) leptin receptor isoform mRNAs. Comp Biochem Physiol 148, 167-173.

Kling P, I Ronnestad, SO Stefansson, K Murashita, T Kurokawa, BJ Bjornsson. 2009. A homologous salmonid leptin radioimmunoassay indicates elevated plasma leptin levels during of rainbow trout. Gen Comp Endocrinol 162, 307-312.

Laemmli UK. 1970. Cleavage of structural proteins during the assembly of the head of bacteriophage T4. Nature 227, 680-685.

Magistrelli D, PG Dimel, F Rosi. 2008. Leptin, insulin and ghrelin levels in goat milk and in plasma of suckling kids. Small Rumin Res 79, 38-41.

Matteri RL, DA Papkoff, JR Swedlow, Y-S Chang. 1986. Isolation and characterization of three forms of luteinizing hormone from the pituitary gland of the horse. Biol Reprod 34, 571-578.

Nilsson J, S Stahl, J Lundeberg, M Uhlen, P Nygren. 1997. Affinity fusion strategies for detection, purification, and immobilization of recombinant proteins. Review. Protein Expression and Purification $11,1-16$.

Porstmann B, T Porstmann, E Nugel, U Evers. 1985. Which of the commonly used marker enzymes gives the best results in colorimetric and fluorimetric enzyme immunoassays: horseradish peroxidase, alkaline phosphatase or beta-galactosidase? J Immunol Methods $10,79,27-37$. 
